Bundesgesundheitsbl $2013 \cdot 56: 749-754$

DOI 10.1007/s00103-013-1690-9

Online publiziert: 27. Mai 2013

c) Springer-Verlag Berlin Heidelberg 2013

\section{Additional material online}

An English full-text version of this article is available at SpringerLink under supplementary material: dx.doi.org/10.1007/s00103-013-1690-9

\section{Hintergrund und Fragestellung}

Eine Stressreaktion ist eine normale Reaktion auf verschiedene Reize und Belastungen. Sie dient der Mobilisierung von Energie, einer verstärkten Blutzirkulation im Gehirn und in den Muskeln und erhöht die Aufmerksamkeit [1]. In Industrie- und Dienstleistungsgesellschaften ist der menschliche Organismus zunehmend komplexen Belastungen in der Arbeits- und Lebenswelt ausgesetzt, auf die er reagieren muss. Laut einer von der Techniker Krankenkasse in Auftrag gegebenen Studie empfinden 8 von 10 Deutschen ihr Leben als stressbelastet, und jeder Dritte leidet unter „Dauerstress" [2].

Wenn die Häufigkeit und Intensität von Stressbelastungen die vorhandenen individuellen Ressourcen zur Stressbewältigung übersteigen, kann es $\mathrm{zu}$ einer chronischen Überforderung in Form von chronischem Stress kommen. Chronischer Stress hat Effekte auf den Stoffwechsel, das Immun- und kardiovaskuläre System und beeinträchtigt die Schlafregulierung, Lern-, Gedächtnis- und Aufmerksamkeitsprozesse [1,2, 3]. Stress scheint auch ein Faktor beim Entstehen und Fortschreiten von psychischen Auffälligkeiten und Störungen zu sein. Bis vor ca. 10 Jahren war hier aufgrund mangelnder Längsschnittstudien aber keine eindeutige Zuordnung von

U. Hapke - U.E. Maske · C. Scheidt-Nave · L. Bode · R. Schlack · M.A. Busch

Abteilung für Epidemiologie und Gesundheitsmonitoring, Robert Koch-Institut, Berlin

\title{
Chronischer Stress bei Erwachsenen in Deutschland
}

\section{Ergebnisse der Studie zur Gesundheit Erwachsener in Deutschland (DEGS1)}

Ursache und Wirkung möglich [1]. Inzwischen gilt die Assoziation zwischen stressbelasteten Lebensereignissen und Episoden einer Major Depression als belegt [4]. Eine umfangreiche Analyse der Literatur zu psychosozialem Stress im Arbeitsumfeld, die auch Längsschnittstudien einschloss, identifizierte Risikofaktoren für die Entwicklung mentaler Störungen [5]. Psychosozialer Dauerstress birgt besonders hohe Risiken für stressbedingte chronische Veränderungen und deren Wechselwirkungen, die meist den bei vorzeitiger Alterung ablaufenden Prozessen entsprechen, wie mit Blick auf das Immunsystem gezeigt werden konnte [6].

Die Untersuchung von chronischem Stress in DEGS1 hat das Ziel, lang andauernde oder häufig wiederkehrende Alltagsbelastungen und ihre Auswirkungen auf die Gesundheit und das psychische Wohlbefinden näher zu untersuchen. Hierbei wird zunächst die Prävalenz von chronischem Stress bei Frauen und Männern in unterschiedlichen Altersgruppen und bei Menschen mit unterschiedlichem sozioökonomischem Status beschrieben. Darüber hinaus werden Zusammenhänge zwischen der Prävalenz von chronischem Stress und sozialer Unterstützung, Schlafstörungen, depressiven Symptomen und dem sog. Burnout-Syndrom untersucht.

\section{Methoden \\ Studienpopulation von \\ DEGS1 und Statistik}

Die „Studie zur Gesundheit Erwachsener in Deutschland" (DEGS) ist Bestandteil des Gesundheitsmonitorings des Robert Koch-Instituts (RKI). Konzept und Design von DEGS sind an anderer Stelle ausführlich beschrieben [7, 8, 9, 10, 11]. Die erste Erhebungswelle (DEGS1) wurde von 2008 bis 2011 durchgeführt und umfasste Befragungen, Untersuchungen und Tests $[12,13]$. Zielpopulation war die in Deutschland lebende Bevölkerung im Alter von 18 bis 79 Jahren.

DEGS1 hat ein Mischdesign, das sowohl quer- als auch längsschnittliche Analysen ermöglicht. Hierbei wurde eine Einwohnermeldeamtsstichprobe gezogen, die die ehemaligen Teilnehmerinnen und Teilnehmer des BundesGesundheitssurveys 1998 (BGS98) ergänzt. Insgesamt nahmen 8152 Personen teil, darunter 4193 Ersteingeladene (Response 42\%) und 3959 ehemalige Teilnehmerinnen und Teilnehmer des BGS98 (Response 62\%). 7238 Personen besuchten eines der 180 Untersuchungszentren, 914 wurden ausschließlich befragt. Die Nettostichprobe ermöglicht für den Altersbereich von 18 bis 79 Jahren $(n=7988$, davon 7116 in Untersuchungszentren) repräsentative 
Tab. 1 Prävalenz starker Belastung durch chronischen Stress unterteilt nach Geschlecht Altersgruppen und sozioökonomischem Status (SES), $\mathrm{N}=5793$

\begin{tabular}{|c|c|c|c|c|c|}
\hline & & $\begin{array}{l}18 \text { bis } \\
29 \text { Jahre } \\
\%(95 \%-K I)\end{array}$ & $\begin{array}{l}30 \text { bis } \\
44 \text { Jahre } \\
\%(95 \%-K I)\end{array}$ & $\begin{array}{l}45 \text { bis } \\
64 \text { Jahre } \\
\% \text { (95\%-KI) }\end{array}$ & $\begin{array}{l}\text { Gesamt } \\
\%(95 \%-K I)\end{array}$ \\
\hline \multirow[t]{4}{*}{ Frauen $(\mathrm{N}=3078)$} & Niedriger SES & $\begin{array}{l}18,0 \\
(11,5-27,1)\end{array}$ & $\begin{array}{l}22,2 \\
(13,3-34,6)\end{array}$ & $\begin{array}{l}20,8 \\
(14,4-29)\end{array}$ & $\begin{array}{l}20,2 \\
(15,9-25,3)\end{array}$ \\
\hline & Mittlerer SES & $\begin{array}{l}16,3 \\
(12,0-21,7)\end{array}$ & $\begin{array}{l}12,9 \\
(9,7-17)\end{array}$ & $\begin{array}{l}11,4 \\
(9,2-14,1)\end{array}$ & $\begin{array}{l}13,0 \\
(11,3-14,9)\end{array}$ \\
\hline & Hoher SES & $\begin{array}{l}12,3 \\
(6,3-22,7)\end{array}$ & $\begin{array}{l}12,4 \\
(8,6-17,5)\end{array}$ & $\begin{array}{l}10,1 \\
(6,9-14,6)\end{array}$ & $\begin{array}{l}11,3 \\
(8,9-14,4)\end{array}$ \\
\hline & Gesamt & $\begin{array}{l}16,1 \\
(12,8-20,1)\end{array}$ & $\begin{array}{l}14 \\
(11,3-17,3)\end{array}$ & $\begin{array}{l}12,6 \\
(10,6-15,0)\end{array}$ & $\begin{array}{l}13,9 \\
(12,3-15,6)\end{array}$ \\
\hline \multirow[t]{4}{*}{$\begin{array}{l}\text { Männer } \\
(\mathrm{N}=2715)\end{array}$} & Niedriger SES & $\begin{array}{l}17,3 \\
(10,0-28,2)\end{array}$ & $\begin{array}{l}13,5 \\
(7,4-23,3)\end{array}$ & $\begin{array}{l}13,4 \\
(8,5-20,6)\end{array}$ & $\begin{array}{l}14,6 \\
(10,9-19,1)\end{array}$ \\
\hline & Mittlerer SES & $\begin{array}{l}8,7 \\
(5,8-12,7)\end{array}$ & $\begin{array}{l}6,9 \\
(4,3-11,0)\end{array}$ & $\begin{array}{l}7,8 \\
(5,8-10,4)\end{array}$ & $\begin{array}{l}7,8 \\
(6,2-9,7)\end{array}$ \\
\hline & Hoher SES & $\begin{array}{l}3,8 \\
(0,8-15,6)\end{array}$ & $\begin{array}{l}4,4 \\
(2,0-9,4)\end{array}$ & $\begin{array}{l}44,7 \\
(3,0-7,2)\end{array}$ & $\begin{array}{l}4,4 \\
(2,9-6,8)\end{array}$ \\
\hline & Gesamt & $\begin{array}{l}9,8 \\
(7,1-13,4)\end{array}$ & $\begin{array}{l}7,1 \\
(5,0-10,0)\end{array}$ & $\begin{array}{l}8,0 \\
(6,5-9,9)\end{array}$ & $\begin{array}{l}8,2 \\
(6,9-9,6)\end{array}$ \\
\hline \multirow[t]{4}{*}{$\begin{array}{l}\text { Gesamt } \\
(\mathrm{N}=5793)\end{array}$} & Niedriger SES & $\begin{array}{l}17,7 \\
(12,5-24,3)\end{array}$ & $\begin{array}{l}17,7 \\
(12,0-25,2)\end{array}$ & $\begin{array}{l}16,9 \\
(12,7-22,1)\end{array}$ & $\begin{array}{l}17,3 \\
(14,6-20,4)\end{array}$ \\
\hline & Mittlerer SES & $\begin{array}{l}12,3 \\
(9,8-15,3)\end{array}$ & $\begin{array}{l}10,1 \\
(8,0-12,6)\end{array}$ & $\begin{array}{l}9,7 \\
(8,2-11,5)\end{array}$ & $\begin{array}{l}10,4 \\
(9,3-11,7)\end{array}$ \\
\hline & Hoher SES & $\begin{array}{l}8,0 \\
(4,3-14,4)\end{array}$ & $\begin{array}{l}7,9 \\
(5,3-11,7)\end{array}$ & $\begin{array}{l}7,2 \\
(5,4-9,6)\end{array}$ & $\begin{array}{l}7,6 \\
(6,0-9,6)\end{array}$ \\
\hline & Gesamt & $\begin{array}{l}12,9 \\
(10,8-15,4)\end{array}$ & $\begin{array}{l}10,5 \\
(8,7-12,6)\end{array}$ & $\begin{array}{l}10,3 \\
(9,0-11,8)\end{array}$ & $\begin{array}{l}11 \\
(10,0-12,1)\end{array}$ \\
\hline
\end{tabular}

Tab. 2 Prävalenz starker Belastung durch chronischen Stress unterteilt nach Geschlecht und sozialer Unterstützung, $\mathrm{N}=5774$

\begin{tabular}{|llll}
\hline & $\begin{array}{l}\text { Geringe soziale Unter- } \\
\text { stützung } \\
\%(95 \%-K I)\end{array}$ & $\begin{array}{l}\text { Mittlere soziale } \\
\text { Unterstützung } \\
\%(95 \%-K I)\end{array}$ & $\begin{array}{l}\text { Starke soziale } \\
\text { Unterstützung } \\
\%(95 \%-K I)\end{array}$ \\
\hline Frauen $(\mathrm{N}=3067)$ & $32,5(25,3-40,5)$ & $13,3(11,3-15,7)$ & $9,5(7,6-11,7)$ \\
\hline Männer $(\mathrm{N}=2707)$ & $20,8(15,2-27,7)$ & $8,4(6,6-10,5)$ & $4,3(2,9-6,3)$ \\
\hline Gesamt $(\mathrm{N}=5774)$ & $26,2(21,6-31,4)$ & $10,8(9,4-12,3)$ & $7(5,7-8,5)$ \\
\hline
\end{tabular}

Querschnittanalysen und Trendaussagen im Vergleich mit dem BGS98 [11]. Die Querschnitt- und Trendanalysen werden mit einem Gewichtungsfaktor durchgeführt, der Abweichungen der Stichprobe von der Bevölkerungsstruktur (Stand 31.12.2010) hinsichtlich Alter, Geschlecht, Region und Staatsangehörigkeit sowie Gemeindetyp und Bildung korrigiert [11]. Bei der Berechnung der Gewichtung für die ehemaligen Teilnehmenden des BGS98 wurde die Wiederteilnahmewahrscheinlichkeit, basierend auf einem logistischen Modell, berücksichtigt. Eine Nonresponder-Analyse und der Vergleich einzelner erhobener Indikatoren mit Daten der amtlichen Statistik weisen auf eine hohe Repräsen- tativität der Stichprobe für die Wohnbevölkerung in Deutschland hin [11].

Um sowohl die Gewichtung als auch die Korrelation der Teilnehmenden innerhalb einer Gemeinde zu berücksichtigen, wurden die Konfidenzintervalle und p-Werte mit den SPSS-20-Verfahren für komplexe Stichproben bestimmt. Unterschiede werden als statistisch signifikant angesehen, wenn sich die jeweiligen 95\%-Konfidenzintervalle nicht überschneiden. Wenn sich die $95 \%$-Konfidenzintervalle nur geringfügig überschneiden wurde die statistische Signifikanz von Prävalenzunterschieden anhand eines korrigierten Chi-Quadrat-Tests auf Unabhängigkeit nach RaoScott [14] ermittelt. Hierbei wurde ein p-
Wert $<0,05$ als statistisch signifikant angesehen.

\section{Chronischer Stress}

Zur Messung der Stressbelastung wurde die Screening-Skala des Trierer Inventars zum chronischen Stress (TICS-SSCS) genutzt [15]. Dieser aus 12 Items bestehende Fragebogen wurde den Altersgruppen bis einschließlich 64 Jahre zur Beantwortung vorgelegt $(\mathrm{N}=5850)$. Die Auswertungen in diesem Beitrag bleiben also auf die Teilnehmerinnen und Teilnehmer der Altersgruppe von 18 bis 64 Jahren begrenzt. Mit dem TICS-SSCS wird die Häufigkeit an subjektiv erlebter Stressbelastung in 5 verschiedenen Stressbereichen in den letzten 3 Monaten erhoben: chronische Besorgnis, arbeitsbezogene und soziale Überlastung, Überforderung und Mangel an sozialer Anerkennung. Die Häufigkeit der Belastung in den 5 Stressbereichen wird jeweils mit den Ausprägungen „nie“ (0 Punkte), „selten“ (1 Punkt), „manchmal“ (2 Punkte), "häufig" (3 Punkte) und „sehr häufig“ (4 Punkte) erfasst. Auf Basis der 12 Itemwerte wurde ein Summenscore berechnet; dabei durften für bis zu 3 Items Antworten fehlen. Der Wertebereich des Summenscores variiert zwischen 0 und 48 Punkten, wobei der Punktwert 0 für niemals Stress und der Punktwert 48 für sehr häufigen Stress in allen 5 Stressbereichen steht. Für $\mathrm{n}=5802$ lag ein Punktwert für den Summenscore subjektiver Stressbelastung vor. Basierend auf der Verteilung der Punktwerte des Summenscores in der Gesamtstichprobe wurden die folgenden Kategorien von Stressbelastung gebildet: 0 bis 11 Punkte ( $\leq \mathrm{Me}$ dian) „unterdurchschnittlich bis durchschnittlich“, 12 bis 22 Punkte ,überdurchschnittlich“, 23 bis 48 Punkte ( $\geq 90$. Perzentil) „stark“.

\section{Burnout-Syndrom}

Zum Burnout-Syndrom wurde im ärztlichen, computergestützten Interview (CAPI) gefragt, ob es jemals von einem Arzt oder Psychotherapeuten festgestellt wurde. Wenn diese Frage bejaht wurde, wurde zusätzlich gefragt, wann es das erste Mal festgestellt wurde, ob es in den letzten 
12 Monaten bestand und welche Behandlungen in Anspruch genommen wurden.

\section{Depressive Symptomatik}

Das Vorliegen einer aktuellen depressiven Symptomatik in den letzten 2 Wochen wurde im Selbstausfüllfragebogen mit dem Depressionsmodul des Patient Health Questionnaire (PHQ) erfasst [16]. Das als PHQ-9 bezeichnete Depressionsmodul ist ein Selbstbeurteilungsinstrument, mit dem das Vorliegen und die Häufigkeit von 9 depressiven Symptomen innerhalb der letzten 2 Wochen gemäß den diagnostischen Kriterien einer Major Depression nach DSM-IV erfragt wird $[16,17]$. Jedes Symptom wird dabei mit den Ausprägungen „Überhaupt nicht“ (0 Punkte), „An einzelnen Tagen“ (1 Punkt), „An mehr als der Hälfte der Tage“ (2 Punkte) oder „Beinahe jeden Tag" (3 Punkte) erfasst. Der Skalensummenwert entspricht der Summe der Punktwerte aller 9 Symptome und variiert zwischen 0 und 27 Punkten. Ein Punktwert von 10 oder mehr Punkten wird als aktuelle depressive Symptomatik definiert $[16,17,18]$.

\section{Schlafstörungen}

Aktuelle Schlafstörungen, definiert als Einschlaf- oder Durchschlafprobleme, wurden als Selbstangabe für den Zeitraum der letzten 4 Wochen erfasst [19].

\section{Bestimmung von Kovariablen}

Der sozioökonomische Status wurde anhand eines Indexes bestimmt, in den Angaben zu schulischer und beruflicher Ausbildung, beruflicher Stellung sowie Haushaltsnettoeinkommen (bedarfsgewichtet) eingehen und der eine Einteilung in niedrige, mittlere und hohe Statusgruppe ermöglicht [20]. Mittels der Oslo-3 Social Support Scale, einem international eingesetzten Drei-Item-Instrument, wurde die wahrgenommene soziale Unterstützung im privaten Umfeld erhoben [21]. Auf Basis des Summenscores wurden die Ausprägungen „geringe Unterstützung “ (3 bis 8 Punkte), „mittlere Unterstützung“ (9 bis 11 Punkte), „starke Unterstützung“ (12 bis 14 Punkte) gebildet [20].

Bundesgesundheitsbl 2013 · 56:749-754 DOI 10.1007/s00103-013-1690-9

(c) Springer-Verlag Berlin Heidelberg 2013

U. Hapke · U.E. Maske · C. Scheidt-Nave · L. Bode · R. Schlack · M.A. Busch

Chronischer Stress bei Erwachsenen in Deutschland. Ergebnisse der Studie zur Gesundheit Erwachsener in Deutschland (DEGS1)

\section{Zusammenfassung}

Die Studie zur Gesundheit Erwachsener in Deutschland (DEGS1) wurde von 2008 bis 2011 durchgeführt und umfasste Befragungen, Untersuchungen und Tests. Zielpopulation war die in Deutschland lebende Bevölkerung im Alter von 18 bis 79 Jahren. Insgesamt nahmen 8152 Personen teil. Chronischer Stress wurde erfasst, um seine Auswirkungen auf die Gesundheit und das psychische Wohlbefinden zu untersuchen. Zur Messung der Stressbelastung wurde die Screening-Skala des Trierer Inventars zum chronischen Stress der Altersgruppe bis einschließlich 64 Jahre zur Beantwortung vorgelegt ( $\mathrm{N}=5850)$. Frauen geben mit 13,9\% signifikant häufiger eine starke Stressbelastung an als Männer (8,2\%). Die Prävalenz starker Stressbelastung nimmt mit steigendem sozioökonomischem Status $\mathrm{ab}$; sie fällt von $17,3 \%$ bei niedrigem auf 7,6\% bei hohem sozioökonomischem Status. Eine starke Belastung mit chronischem Stress ist besonders häufig $(26,2 \%)$, wenn eine geringe soziale Unterstützung vorliegt. Menschen mit einer starken Belastung durch chronischen Stress zeigen deutlich häufiger eine depressive Symptomatik, ein Burnout-Syndrom oder Schlafstörungen als Menschen ohne starke Belastung durch chronischen Stress. Die Bedeutung von chronischem Stress als Gesundheitsrisiko wird durch die Daten bestätigt. Das Thema hat somit eine hohe PublicHealth-Relevanz.

\section{Schlüsselwörter}

Stressbelastung · Burnout-Syndrom .

Depression · Schlafstörungen ·

Gesundheitssurvey

\section{Chronic stress among adults in Germany. Results of the German Health Interview and Examination Survey for Adults (DEGS1)}

\section{Abstract}

The German Health Interview and Examination Survey for Adults (DEGS1) was conducted from 2008-2011 and comprised interviews, examinations and tests. The target population was the resident population of Germany aged 18-79 years. A total of 8,152 persons participated. Chronic stress was assessed to examine its effects on health and mental wellbeing. The Screening Scale of the Trier Inventory for the Assessment of Chronic Stress was used to assess stress burden among participants up to the age of 64 years $(N=5,850)$. High levels of stress are significantly more often reported by women (13.9\%) than by men (8.2\%). The prevalence of high stress levels decreases with a higher socioeconomic status (SES); it falls from $17.3 \%$ with low SES to $7.6 \%$ with high SES. High chronic stress levels are particularly common (26.2\%) in persons who report low levels of social support. Depressive symptoms, burnout syndrome and sleep disturbances are more common in people who have high levels of chronic stress than in those without high levels of stress. The results confirm the importance of chronic stress as a health risk and underline the public health relevance of chronic stress. An English full-text version of this article is available at SpringerLink as supplemental.

\section{Keywords}

Stress burden - Burnout syndrome . Depression · Sleep disturbances · Health survey

\section{Ergebnisse}

Die Prävalenz starker Stressbelastung nach Alter, Geschlecht und sozioökonomischem Status ist in • Tab. 1 dargestellt. Frauen geben mit 13,9\% signifikant häufiger eine überdurchschnittliche Stressbelastung an als Männer (8,2\%). Zwischen den Altersgruppen gibt es keine signifikanten Unterschiede. Die Prävalenz starker Stressbelastung nimmt insgesamt mit steigendem sozioökonomischem Status ab; sie fällt von $17,3 \%$ bei niedrigem auf 7,6\% bei hohem sozioökonomischem Status. Bei den Frauen ist der Unterschied zwischen mittlerem und hohem SES nicht signifikant $(\mathrm{p}=0,326)$. 


\begin{tabular}{|c|c|c|c|c|c|c|c|c|c|}
\hline & \multicolumn{3}{|c|}{ Burnout-Syndrom i.d.I. 12 Monaten } & \multicolumn{3}{|c|}{ Aktuelle depressive Symptomatik } & \multicolumn{3}{|c|}{ Einschlaf- oder Durchschlafprobleme } \\
\hline & $N$ & $\begin{array}{l}\text { Vorhanden } \\
\%(95 \%-\mathrm{KI})\end{array}$ & $\begin{array}{l}\text { Nicht vorhanden } \\
\%(95 \%-\mathrm{KI})\end{array}$ & $N$ & $\begin{array}{l}\text { Vorhanden } \\
\%(95 \%-\mathrm{KI})\end{array}$ & $\begin{array}{l}\text { Nicht vorhanden } \\
\%(95 \%-\mathrm{KI})\end{array}$ & $N$ & $\begin{array}{l}\text { Vorhanden } \\
\%(95 \%-\mathrm{KI})\end{array}$ & $\begin{array}{l}\text { Nicht vorhanden } \\
\%(95 \%-\mathrm{KI})\end{array}$ \\
\hline Frauen & 3044 & $\begin{array}{l}51,5 \\
(37,3-65,4)\end{array}$ & $\begin{array}{l}12,9 \\
(11,5-14,6)\end{array}$ & 2987 & $\begin{array}{l}57,7 \\
(49,8-65,2)\end{array}$ & $\begin{array}{l}8,5 \\
(7,3-9,8)\end{array}$ & 3007 & $\begin{array}{l}24,4 \\
(20,7-28,6)\end{array}$ & $\begin{array}{l}9,7 \\
(8,3-11,4)\end{array}$ \\
\hline Männer & 2705 & $\begin{array}{l}36,1 \\
(20,2-55,9)\end{array}$ & $\begin{array}{l}7,8 \\
(6,5-9,2)\end{array}$ & 2654 & $\begin{array}{l}47,4 \\
(39,2-55,7)\end{array}$ & $\begin{array}{l}5,4 \\
(4,3-6,7)\end{array}$ & 2678 & $\begin{array}{l}18,9 \\
(15,0-23,5)\end{array}$ & $\begin{array}{l}5,4 \\
(4,3-6,9)\end{array}$ \\
\hline Gesamt & 5749 & $\begin{array}{l}45,9 \\
(35,2-57,1)\end{array}$ & $\begin{array}{l}10,3 \\
(9,4-11,3)\end{array}$ & 5641 & $\begin{array}{l}53,7 \\
(48,2-59,2)\end{array}$ & $\begin{array}{l}6,9 \\
(6,1-7,7)\end{array}$ & 5685 & $\begin{array}{l}22,1 \\
(19,3-25,2)\end{array}$ & $\begin{array}{l}7,5 \\
(6,6-8,4)\end{array}$ \\
\hline
\end{tabular}

\section{Starke Stressbelastung und soziale Unterstützung}

Eine starke Belastung mit chronischem Stress ist besonders häufig (26,2\%), wenn eine geringe soziale Unterstützung vorliegt (• Tab.2). Das trifft sowohl auf Männer als auch auf Frauen zu. Umgekehrt sinkt bei starker sozialer Unterstützung die Häufigkeit starker Stressbelastung signifikant auf ein viel niedrigeres Niveau (7\%).

\section{Starke Stressbelastung und psychische Beeinträchtigungen}

Menschen mit einer starken Belastung durch chronischen Stress zeigen deutlich häufiger eine aktuelle depressive Symptomatik, ein Burnout-Syndrom oder Schlafstörungen als Menschen ohne starke Belastung durch chronischen Stress. Das gilt sowohl für Männer als auch für Frauen. Mehr als jeder zweite Erwachsene mit aktueller depressiver Symptomatik fühlt sich durch chronischen Stress stark belastet (53,7\%). Dies gilt ebenso für knapp jede zweite Person (45,9\%), bei der ein Burnout-Syndrom diagnostiziert wurde und trifft immerhin bei jedem Fünften (22,1\%) mit Schlafstörungen $\mathrm{zu}(\bullet$ Tab. 3).

\section{Starke Stressbelastung \\ und psychische \\ Mehrfachbeeinträchtigungen}

In - Abb. 1 zeigt sich, dass Beeinträchtigungen durch Burnout, durch eine depressive Symptomatik oder durch Schlafstörungen mit steigender Stressbelastung zunehmen. Personen mit unterdurchschnittlicher bis durchschnittlicher Stressbelastung sind $\mathrm{zu} 16,4 \%$ von solchen Beeinträchtigungen betroffen; bei Menschen mit starker Stressbelastung steigt dieser Wert hingegen auf 61,1\%. Gleichzeitig nimmt mit steigender Stressbelastung auch die Prävalenz von Mehrfachbelastungen durch 2 oder 3 psychische Beeinträchtigungen zu. Insgesamt fallen die Beeinträchtigungen bei starker Stressbelastung bei Frauen etwas höher aus.

\section{Diskussion}

In der ersten Welle von DEGS wurde subjektiver chronischer Stress erstmalig bei 5802 Probanden im Alter von 18 bis 64 Jahren mit einem standardisierten Instrument erfasst (TICS) [15]. Die verwendete Screening-Version (SSCS) deckt dabei 5 miteinander in Beziehung stehende Dimensionen von chronischem Stress ab. Mit TICS wurde ein hinsichtlich Faktorenstruktur und psychometrischer Eigenschaften für Populationsstudien validiertes Instrument verwendet [22].

In DEGS1 wurden gesundheitliche Effekte von chronischem Stress fokussiert auf psychische Beeinträchtigungen untersucht. Die Ergebnisse belegen eindrücklich einen hohen Zusammenhang zwischen Belastungen durch chronischen Stress und psychischen Beeinträchtigungen durch depressive Symptome, Burnout-Syndrom und Schlafstörungen. In unserer Stichprobe nimmt das Auftreten von chronischem Stress mit steigendem sozioökonomischem Status (SES) ab. In der Stichprobe, die der Entwicklung des Tests diente, waren die Werte der Scree-
ning-Skala zum chronischen Stress nicht signifikant mit der Schulbildung korreliert [15]. Zukünftige vertiefende Analysen unserer Daten werden vermutlich Aufschluss darüber geben können, welche spezifischen SES-Aspekte mit einer geringeren Stressbelastung einhergehen.

Die Ergebnisse zur höheren Stressbelastung von Frauen und von Personen in jüngeren Altersgruppen sind konsistent mit den Ergebnissen, die in der Testpublikation veröffentlicht wurden [15]. Bezüglich der sozialen Unterstützung konnten die in der Testpublikation berichteten Unterschiede bestätigt werden. Soziale Unterstützung fungiert gemäß der Interpretation der Testautoren als Ressource, die bei chronischem Stress der Bewältigung dient und als Puffer wirkt.

Mit Blick auf die geminderte Schlafqualität bei chronischem Stress lassen sich unsere Ergebnisse vermutlich kausal interpretieren. In einer entsprechenden Längsschnittuntersuchung konnte der Einfluss von chronischem Stress auf die Schlafqualität aufgezeigt werden, aber umgekehrt kein Einfluss der Schlafqualität auf die subjektive Wahrnehmung von chronischem Stress [23].

Der Zusammenhang zwischen chronischem Stress und psychischen Beschwerden, insbesondere mit depressiven Symptomen, ist konsistent zu früheren $\mathrm{Be}$ funden [15]. Hier ist anzumerken, dass dieser Zusammenhang durch die Überschneidung einzelner Fragebogeninhalte im PHQ-9 und TICS-SSCS verstärkt werden kann [15]. Ein Beispiel ist das Item „Zeiten, in denen ich mir viele Sorgen mache und damit nicht aufhören kann" des SSCS. Teilnehmerinnen und Teilneh- 


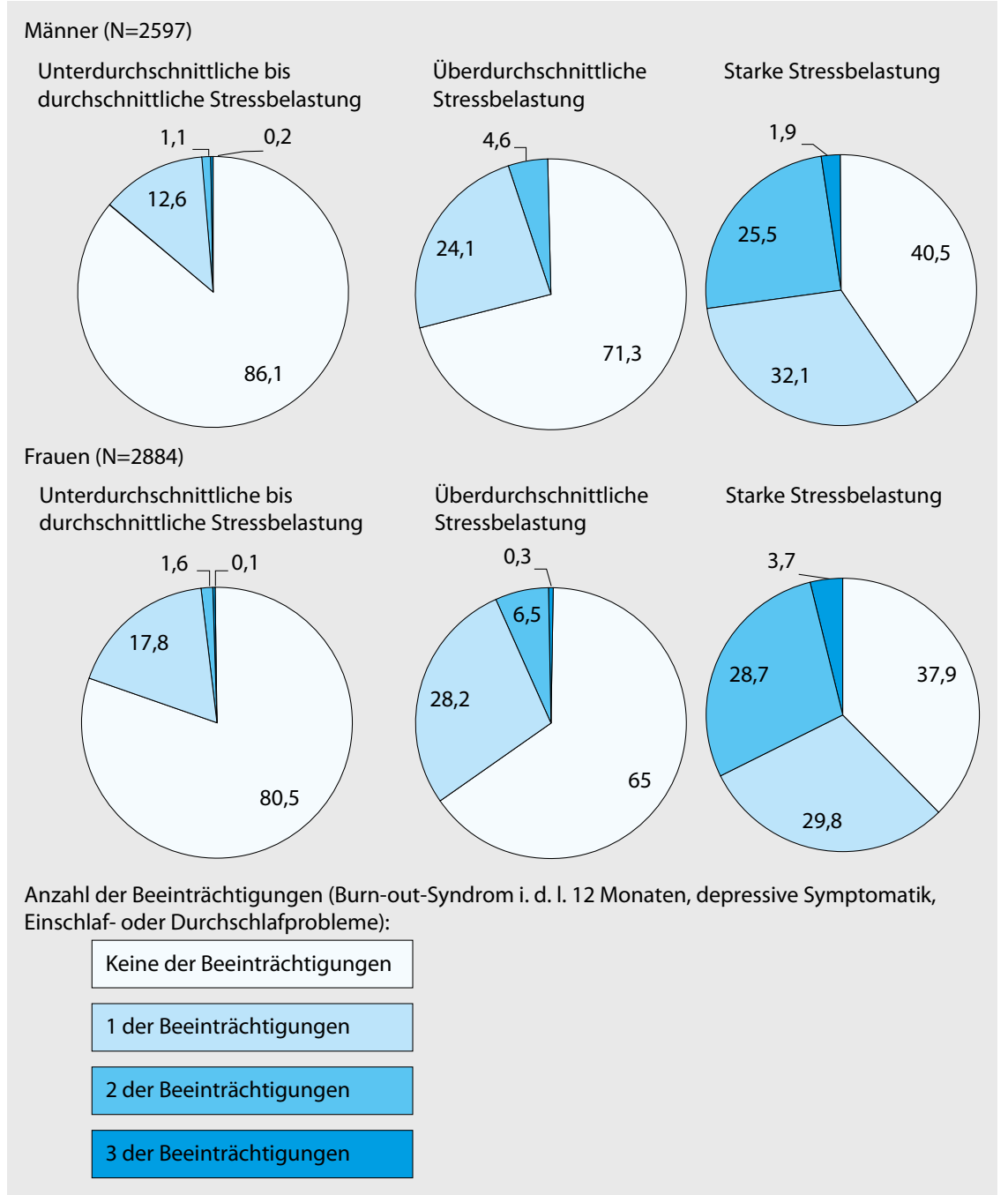

Abb. 1 A Prävalenz psychischer Beeinträchtigungen in Abhängigkeit von Stressbelastung, N=5481

mer, die hier „sehr häufig“ angeben, haben zu $61 \%$ eine aktuelle depressive Symptomatik gemäß PHQ-9. Aber auch insgesamt war die Prävalenz einer depressiven Symptomatik bei starker Stressbelastung hoch (Prävalenz 53,7\%). In einer australischen Stichprobe von Frauen konnte gezeigt werden, dass sowohl chronischer Stress (6 Monate) als auch akuter Stress mit dem Beginn einer depressiven Episode assoziiert ist [24].

Als Burnout-Syndrom wird ein $\mathrm{Zu}-$ stand emotionaler Erschöpfung, Depersonalisation und reduzierter Leistungsfähigkeit bezeichnet, der aufgrund beruflicher stressbedingter Überlastung entstanden ist. Insoweit ist das BurnoutSyndrom sehr eng mit dem Stressbegriff im Sinne von Arbeitsstress verknüpft. Die Differenzialdiagnostik des Burnout-
Syndroms ist bis heute nicht standardisiert, was die diesbezügliche Vergleichbarkeit von Studien erschwert [25]. Dass in unserer Studie bei fast jedem zweiten Probanden mit einem Burnout-Syndrom eine starke Stressbelastung gefunden wurde, ist nicht überraschend. Von einer Feinanalyse der Arbeitsstress-bezogenen Belastung sind zukünftig differenzierte Erkenntnisse zu erwarten.

Die Bedeutung von psychischen Beeinträchtigungen als Gesundheitsrisiko steht dennoch außer Frage. Gerade konnte in einer Metaanalyse von 10 prospektiven englischen Kohortenstudien mit fast 70.000 Teilnehmern aus der Allgemeinbevölkerung nachgewiesen werden, dass das Mortalitätsrisiko (alle Todesursachen) in Assoziation mit psychosozialem Stress um $21 \%$ steigt [26].
Fazit

Das Ziel, mit DEGS1 besonders von chronischem Stress betroffene Bevölkerungsgruppen zu identifizieren und den Zusammenhang zwischen chronischem Stress und gesundheitlichen Beschwerden und Störungen näher zu untersuchen, ist ein Thema mit hoher Public-Health-Relevanz.

\section{Korrespondenzadresse}

\section{Dr. U. Hapke}

Abteilung für Epidemiologie und Gesundheitsmonitoring, Robert Koch-Institut General-Pape-Str. 62-66, 12101 Berlin U.Hapke@RKI.de

Finanzierung der Studie. Die Studie wurde finanziert mit Mitteln des Robert Koch-Instituts und des Bundesministeriums für Gesundheit.

Interessenkonflikt. Der korrespondierende Autor gibt für sich und seine Koautoren an, dass kein Interessenkonflikt besteht.

\section{Literatur}

1. Kudielka BM, Kirschbaum C (2001) Stress and health research. In: Baltes NJSP (Hrsg) The international encyclodedia of the social and behavioral sciences. Elsevier, Oxford

2. TK (2009) Stress - Aktuelle Bevölkerungsbefragung: Ausmaß, Ursachen und Auswirkungen von Stress in Deutschland. In: F.A.Z.-Institut für Management-, Markt und Medieninformationen GmbH \& Techniker Krankenkasse, Techniker Krankenkasse, Pressestelle, Hamburg. http://www.tkonline.de

3. Chrousos GP (2009) Stress and disorders of the stress system. Nat Rev Endocrinol 5:374-381

4. Hammen C (2005) Stress and depression. Annu Rev Clin Psychol 1:293-319

5. Stansfeld S, Candy B (2006) Psychosocial work environment and mental health - a meta-analytic review. Scand J Work Environ Health 32:443-462

6. Bauer ME, Jeckel CM, Luz C (2009) The role of stress factors during aging of the immune system. Ann N Y Acad Sci 1153:139-152

7. Kurth BM, Lange C, Kamtsiuris P, Hölling H (2009) Gesundheitsmonitoring am Robert Koch-Institut. Sachstand und Perspektiven. Bundesgesundheitsbl Gesundheitsforsch Gesundheitsschutz 52:557-570

8. Kurth BM (2012) Das RKI-Gesundheitsmonitoring - was es enthält und wie es genutzt werden kann. Public Health Forum 20(76):4.e1-4.e3

9. Gößwald A, Lange M, Kamtsiuris P, Kurth BM (2012) DEGS: Studie zur Gesundheit Erwachsener in Deutschland. Bundesweite Quer- und Längsschnittstudie im Rahmen des Gesundheitsmonitorings des Robert Koch-Instituts. Bundesgesundheitsbl Gesundheitsforsch Gesundheitsschutz 55:775-780 


\section{Leitthema}

10. Scheidt-Nave $C$, Kamtsiuris $P, G o ̈ ß w a l d ~ A$ et al (2012) German Health Interview and Examination Survey for Adults (DEGS) - design, objectives and implementation of the first data collection wave. BMC Public Health 12:730

11. Kamtsiuris $P$, Lange M, Hoffmann R et al (2013) Die erste Welle der Studie zur Gesundheit Erwachsener in Deutschland (DEGS1). Stichprobendesign Response, Gewichtung und Repräsentativität. Bundesgesundheitsbl Gesundheitsforsch Gesundheitsschutz 56:620-630

12. Gößwald A, Lange M, Dölle R, Hölling H (2013) Die erste Welle der Studie zur Gesundheit Erwachsener in Deutschland (DEGS1). Gewinnung von Studienteilnehmenden, Durchführung der Feldarbeit und Qualitätsmanagement. Bundesgesundheitsbl Gesundheitsforsch Gesundheitsschutz 56:611-619

13. Robert Koch-Institut (Hrsg) (2009) DEGS: Studie zur Gesundheit Erwachsener in Deutschland - Projektbeschreibung. Beiträge zur Gesundheitsberichterstattung des Bundes. RKI, Berlin

14. Rao JNK, Scott AJ (1981) The analysis of categorical data from complex samples surveys: chi-squared tests for goodness of fit and independence in twoway tables. J Am Stat Assoc 76:221-230

15. Schulz P, Schlotz W, Becker P (2004) TICS Trierer Inventar zum chronischen Stress. Hogrefe, Göttingen
16. Löwe B, Spitzer R, Zipfel S, Herzog W (2002) Gesundheitsfragebogen für Patienten (PHQ-D). Komplettversion und Kurzform. Testmappe mit Manual, Fragebögen, Schablonen

17. Busch MA, Maske UE, Ryl L et al (2013) Prävalenz von depressiver Symptomatik und diagnostizierter Depression bei Erwachsenen in Deutschland. Ergebnisse der Studie zur Gesundheit Erwachsener in Deutschland (DEGS1). Bundesgesundheitsbl Gesundheitsforsch Gesundheitsschutz 56:733-739

18. Strine TW, Mokdad AH, Balluz LS et al (2008) Depression and anxiety in the United States: findings from the 2006 behavioral risk factor surveillance system. Psychiatr Serv 59:1383-1390

19. Schlack R, Hapke U, Maske U et al (2013) Häufigkeit und Verteilung von Schlafproblemen und Insomnie in der deutschen Erwachsenenbevölkerung. Ergebnisse der Studie zur Gesundheit Erwachsener in Deutschland (DEGS1). Bundesgesundheitsbl Gesundheitsforsch Gesundheitsschutz 56:740-748

20. Lampert T, Kroll L, Müters S, Stolzenberg H (2013) Messung des sozioökonomischen Status in der Studie zur Gesundheit Erwachsener in Deutschland (DEGS1). Bundesgesundheitsbl Gesundheitsforsch Gesundheitsschutz 56:631-636
21. Dalgard OS, Dowrick C, Lehtinen V et al (2006) Negative life events, social support and gender difference in depression: a multinational community survey with data from the ODIN study. Soc Psychiatry Psychiatr Epidemiol 41:444-451

22. Petrowski K, Paul S, Albani C, Brahler E (2012) Factor structure and psychometric properties of the trier inventory for chronic stress (TICS) in a representative German sample. BMC Med Res Methodol 12:42

23. Schulz P, Hellhammer J, Schlotz W (2003) Arbeitsstress, sozialer Stress und Schlafqualität. Z Gesundheitspsychol 11:1-9

24. Hammen C, Kim EY, Eberhart NK, Brennan PA (2009) Chronic and acute stress and the prediction of major depression in women. Depress Anxiety $26: 718-723$

25. Korczak D, Huber B (2012) Burn-out. Bundesgesundheitsbl Gesundheitsforsch Gesundheitsschutz 55:164-171

26. Russ TC, Stamatakis E, Hamer M et al (2012) Association between psychological distress and mortality: individual participant pooled analysis of 10 prospective cohort studies. BMJ 345:e4933

\section{Hier steht eine Anzeige.}

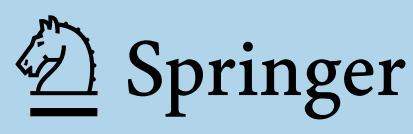

